\title{
ROOTS OF BERNSTEIN-SATO POLYNOMIALS FOR MONOMIAL IDEALS: A POSITIVE CHARACTERISTIC APPROACH
}

\author{
Nero Budur, Mircea Mustaţã, and Morihiko Saito
}

\begin{abstract}
We describe the roots of the Bernstein-Sato polynomial of a monomial ideal using reduction mod $p$ and invariants of singularities in positive characteristic. We give in this setting a positive answer to a problem from [MTW] concerning the dependence on the characteristic for these invariants of singularities.
\end{abstract}

\section{Introduction}

The Bernstein-Sato polynomial (or $b$-function) of an arbitrary ideal in a polynomial ring was introduced in [BMS2] generalizing the case of principal ideals. For monomial ideals, it was shown there that this polynomial can be computed algorithmically in principle, using for example Macaulay2. In this paper we use a positive characteristic approach to give a description of the roots of the Bernstein-Sato polynomial in this case. This is a by-product of positive answers to questions in $[\mathrm{MTW}]$ on the dependence on the characteristic for some invariants of singularities.

In order to explain our approach, we recall the definition of the invariants from $[\mathrm{MTW}]$. Let $\mathfrak{a}$ be a nonzero ideal in a regular local ring $R$ of characteristic $p>0$. Suppose that $J$ is a proper ideal in $R$ whose radical contains a. For every positive integer $e$, let $J^{\left[p^{e}\right]}$ be the ideal generated by the $p^{e}$ th powers of the elements of $J$, and

$$
\nu_{\mathfrak{a}}^{J}\left(p^{e}\right):=\max \left\{\ell \geq 0 \mid \mathfrak{a}^{\ell} \nsubseteq J^{\left[p^{e}\right]}\right\} .
$$

Suppose now that we start with ideals $\mathfrak{a}$ and $J$ in $\mathbb{Z}\left[X_{1}, \ldots, X_{n}\right]$ such that $\mathfrak{a}$ is contained in the radical of $J$, and let $b_{\mathfrak{a}}(s)$ be the Bernstein-Sato polynomial of $\mathfrak{a}$. We consider the invariants associated to the reductions $\bmod p$ of $\mathfrak{a}$ and $J$ around the origin. It was shown in [MTW] that if $p \gg 0$, then $b_{\mathfrak{a}}\left(\nu_{\mathfrak{a}}^{J}\left(p^{e}\right)\right) \equiv 0$ $(\bmod p)$ for all $e$ (see also Proposition 2.2 below).

One expects that in many cases there are polynomial formulas for the invariants $\bmod p$, formulas depending on a suitable congruence of $p$. More precisely, in good situations there should be a positive integer $N$, and for every $i$ relatively

Received by the editors May 23, 2005.

Key words and phrases. Bernstein-Sato polynomials, monomial ideals, reduction mod $p$. 2000 Mathematics Subject Classification. Primary 14B05; Secondary 13N10, 32S40.

The research of the second author was partially supported by NSF grant 0500127 . 
prime to $N$ there should be a polynomial $P_{i} \in \mathbb{Q}[t]$ such that $\nu_{\mathfrak{a}}^{J}\left(p^{e}\right)=P_{i}(p)$ whenever $p$ is large enough and $p \equiv i(\bmod N)$. When this holds, Dirichlet's Theorem on the distribution of prime numbers implies that we get roots of the Bernstein-Sato polynomial over $\mathbb{Q}$ : in fact, each $P_{i}(0)$ is such a root (see Remark 2.4). Our main results are that such formulas can be given for monomial ideals (see Theorem 4.1), and furthermore that all the roots can be obtained in this way (see Theorem 4.9).

The proofs of the above results give in particular a description of all roots of the Bernstein-Sato polynomial of a monomial idea. As a consequence we deduce the following description $\bmod \mathbb{Z}$ of the roots. Suppose that $\mathfrak{a}$ is a proper nonzero ideal in $\mathbb{Z}\left[X_{1}, \ldots, X_{n}\right]$ generated by monomials. The Newton polyhedron $P_{\mathfrak{a}}$ of $\mathfrak{a}$ is the convex hull in $\mathbb{R}_{+}^{n}$ of those $u$ in $\mathbb{N}^{n}$ such that the monomial $X^{u}$ is in $\mathfrak{a}$. For every facet $Q$ of $P_{\mathfrak{a}}$ that is not contained in a coordinate hyperplane, there is a unique linear function $L_{Q}$ on $\mathbb{R}^{n}$ having rational coefficients such that $Q=P_{\mathfrak{a}} \cap L_{Q}^{-1}(1)$. (Here a facet means a maximal-dimensional face.) We denote by $m_{Q}$ the smallest positive integer such that $m_{Q} L_{Q}$ has integer coefficients.

Corollary 1.1. The set consisting of the images in $\mathbb{Q} / \mathbb{Z}$ of the roots of the Bernstein-Sato polynomial of $\mathfrak{a}$ is equal to

$$
\left\{\frac{m}{m_{Q}}+\mathbb{Z} \mid Q \text { facet of } P_{\mathfrak{a}} \text { and } 0 \leq m<m_{Q}\right\} .
$$

One can deduce from our results a description of the roots of the BernsteinSato polynomial, and not just of their classes $\bmod \mathbb{Z}$ (see Remark 4.6). Note that such a description has to be more involved, as the roots do not depend only on the integral closure of the ideal (or equivalently, on the Newton polyhedron). Another, more explicit description of the roots of the Bernstein-Sato polynomial of monomial ideals (with a direct, combinatorial proof) will appear in [BMS1].

A few words about the structure of the paper: in the next section we recall the definition of Bernstein-Sato polynomials and give some details in the general set-up about the connection between their roots and the invariants obtained by reduction mod $p$. In $\S 3$ we specialize to monomial ideals, and give a direct proof of the existence of the Bernstein-Sato polynomial in this case. This will be useful later, as it provides useful information about the roots (see Remark 3.5). The idea is based on the approach to computing Bernstein-Sato polynomials of monomial ideals from [BMS2]. In $\S 4$, we study the invariants of the reduction $\bmod p$ for monomial ideals. We give a polynomial formula for these invariants (depending on a suitable congruence of $p$ ) and show that all the roots of the Bernstein-Sato polynomial can be obtained by our method. The fourth section is devoted to some examples. In the Appendix we show that if $\mathfrak{a}$ is a monomial ideal, it is enough to consider only positive characteristic invariants that correspond to monomial ideals $J$. 


\section{Bernstein-Sato polynomials and reduction $\bmod p$}

We start by recalling some general facts about Bernstein-Sato polynomials. For proofs and details we refer to [BMS2].

Let $\mathfrak{a} \subseteq\left(X_{1}, \ldots, X_{n}\right) \subseteq \mathbb{C}[X]=\mathbb{C}\left[X_{1}, \ldots, X_{n}\right]$ be a nonzero ideal, and let $f_{1}, \ldots, f_{r}$ be nonzero generators of $\mathfrak{a}$. The Bernstein-Sato polynomial $b_{\mathfrak{a}}(s) \in$ $\mathbb{C}[s]$ of $\mathfrak{a}$ is the monic generator of the ideal consisting of those $b(s)$ for which we have a relation

$$
b\left(s_{1}+\ldots+s_{r}\right) \prod_{j} f_{j}^{s_{j}}=\sum_{c} P_{c} \cdot \prod_{j, c_{j}<0}\left(\begin{array}{c}
s_{j} \\
-c_{j}
\end{array}\right) \prod_{j} f_{j}^{s_{j}+c_{j}},
$$

where the above sum is over finitely many $c \in \mathbb{Z}^{r}$ such that $\sum_{j} c_{j}=1$, and where $P_{c} \in \mathbb{C}\left[X, \partial_{X}, s\right]$ for all $c$. (Here $\prod_{j, c_{j}<0}$ means that the product is over the $j$ such that $c_{j}<0$.) As usual, if $m>0$, then the notation $\left(\begin{array}{c}s_{j} \\ m\end{array}\right)$ stands for $\frac{1}{m !} s_{j}\left(s_{j}-1\right) \ldots\left(s_{j}-m+1\right)$.

In (1) one has to interpret the equality formally. Note that if $r=1$, then this equation can be rewritten as

$$
b(s) f^{s}=P\left(X, \partial_{X}, s\right) \cdot f^{s+1},
$$

so we recover the usual definition of the Bernstein-Sato polynomial of a principal ideal (see $[\mathrm{Bj}],[\mathrm{Ka}]$ ).

The fact that there is a nonzero $b(s)$ as above is proved in [BMS2], where one also shows that it does not depend on the choice of generators and that all its roots are negative rational numbers. We refer to [BMS2] also for the motivation for the defining formula (1) in terms of $V$-filtrations.

In our case the ideal is defined over $\mathbb{Z}$, and the $P_{c}$ can be defined over $\mathbb{Q}$ by the following

Proposition 2.1. If the ideal $\mathfrak{a}$ is defined over a subfield $K$ of $\mathbb{C}$, then the $P_{c}$ in (1) can be defined also over $K$.

Proof. There is a finitely generated $K$-subalgebra $A$ of $\mathbb{C}$ such that the $P_{c}$ are defined over $A$. Choosing a maximal ideal of $A$ and taking the image in the corresponding residue field $K^{\prime}$ of $A$, we get a relation in which the $P_{c}$ are defined over a finite extension $K^{\prime}$ of $K$. We may assume that $K^{\prime} / K$ is Galois by enlarging $K^{\prime}$ if necessary. Averaging by the action of the Galois group (where the Galois group acts on the coefficients of $P_{c}$ ), we get the assertion.

From now on, we consider only ideals in $\mathbb{Z}[X]$, as this will be enough for our purpose. We will always work in a neighborhood of the origin. Note that there is a local notion of Bernstein-Sato polynomial, where we require (1) to hold only in some open subset containing the origin. In the rest of this section one could replace $b_{\mathfrak{a}}$ by this local version. However, in what follows we are interested in monomial ideals (which are homogeneous), so in this case there will be no distinction between local and global Bernstein-Sato polynomials. 
If $p$ is a prime number, the ideal $\mathfrak{a}$ defines by reduction $\bmod p$ (and localization) an ideal $\mathfrak{a}_{p}$ in $R_{p}:=\mathbb{F}_{p}[X]_{\left(X_{1}, \ldots, X_{n}\right)}$, where $\mathbb{F}_{p}=\mathbb{Z} / p \mathbb{Z}$ as usual. Recall that if $I$ is an ideal in $R_{p}$ and if $e \geq 1$, then $I^{\left[p^{e}\right]}=\left(g^{p^{e}} \mid g \in I\right)$. To simplify the notation, for $\mathfrak{a} \subseteq \operatorname{Rad}(J) \subseteq\left(X_{1}, \ldots, X_{n}\right)$, we will denote by $\nu_{\mathfrak{a}}^{J}\left(p^{e}\right)$ the largest $\ell$ such that $\mathfrak{a}_{p}^{\ell} \nsubseteq J_{p}^{\left[p^{e}\right]}$.

By Proposition $2.1, b_{\mathfrak{a}}$ and all $P_{c}$ in (1) have coefficients in $\mathbb{Q}$, and hence in $\mathbb{Z}\left[m^{-1}\right]$ for some integer $m$. Moreover, we may assume that for all $c$ that appear in (1) and for all $j$ such that $c_{j}<0,\left(-c_{j}\right)$ ! divides $m$. If $p$ does not divide $m$, then (1) will hold also after reduction $\bmod p$. We will apply this equality by letting $s_{1}, \ldots, s_{r}$ to be nonnegative integers. Note that in this case, if $c_{j}<0$ and $s_{j}+c_{j}<0$, then $\left(\begin{array}{c}s_{j} \\ -c_{j}\end{array}\right)=0$, so the corresponding term in (1) vanishes.

The key to our approach is the following elementary observation from [MTW]. The proof below uses Proposition 2.1, and may be slightly easier than the original one, although both arguments are essentially the same.

Proposition 2.2. Let $\mathfrak{a}$ and $J$ be nonzero ideals in $\mathbb{Z}[X]$ such that $\mathfrak{a} \subseteq \operatorname{Rad}(J) \subseteq$ $\left(X_{1}, \ldots, X_{n}\right)$. Let $m$ be as above. If $p$ does not divide $m$ and $e \geq 1$, then

$$
b_{\mathfrak{a}}\left(\nu_{\mathfrak{a}}^{J}\left(p^{e}\right)\right)=0 \text { in } \mathbb{F}_{p} .
$$

Proof. We can find nonnegative integers $a_{1}, \ldots, a_{r}$ such that $\sum_{j} a_{j}=\nu_{\mathfrak{a}}^{J}\left(p^{e}\right)$ and $\prod_{j} f_{j}^{a_{j}} \notin J_{p}^{\left[p^{e}\right]}$. On the other hand, the hypothesis implies $\prod_{j} f_{j}^{b_{j}} \in J_{p}^{\left[p^{e}\right]}$ if $\sum_{j} b_{j}=\nu_{\mathfrak{a}}^{J}\left(p^{e}\right)+1$ and $b_{j} \geq 0$ for all $j$.

Using (1) $\bmod p$ for $s_{j}=a_{j}$ (together with the remark before this proposition) and the fact that $J_{p}^{\left[p^{e}\right]}$ is an $\mathbb{F}_{p}\left[X, \partial_{X}\right]$-submodule of $\mathbb{F}_{p}[X]$, we deduce that $b_{\mathfrak{a}}\left(\nu_{\mathfrak{a}}^{J}\left(p^{e}\right)\right)=0$ in $\mathbb{F}_{p}$.

This can be used to give roots of the Bernstein-Sato polynomial whenever one can solve the following problem from $[\mathrm{MTW}]$.

Problem 2.3. Under good conditions on $\mathfrak{a}$ and $J$ there should exist a positive integer $N$ together with polynomials $P_{i}$ of degree $e$ in $\mathbb{Q}[t]$ for every $i \in(\mathbb{Z} / N \mathbb{Z})^{\times}$ such that the following holds. If a prime $p$ is sufficiently large and $p \equiv i(\bmod$ $N)$, then $\nu_{\mathfrak{a}}^{J}\left(p^{e}\right)=P_{i}(p)$. Moreover, one should be able to choose $N$ to depend only on $\mathfrak{a}$.

Remark 2.4. Note that if we have $P_{i}$ as in the above problem, then $b_{\mathfrak{a}}\left(P_{i}(p)\right)=$ 0 in $\mathbb{F}_{p}$ for infinitely many primes $p$ by Proposition 2.2 and Dirichlet's Theorem. Therefore $P_{i}(0)$ is a root of $b_{\mathfrak{a}}$. Here we may assume that $P_{i}$ is defined over $\mathbb{Z}\left[m^{-1}\right]$, replacing $m$ by a multiple if necessary. A basic question is which roots can be obtained in this way (Example 4.1 in [MTW] shows that there might be roots which are not detected by the above method). 
We mention that the asymptotic behavior of $\nu_{\mathfrak{a}}^{J}\left(p^{e}\right)$ for $e \rightarrow \infty$ is measured by the $F$-threshold $\mathrm{c}^{J}\left(\mathfrak{a}_{p}\right)$ introduced in [MTW]: this is defined by

$$
\mathrm{c}^{J}\left(\mathfrak{a}_{p}\right):=\lim _{e \rightarrow \infty} \frac{\nu_{\mathfrak{a}}^{J}\left(p^{e}\right)}{p^{e}}=\sup _{e} \frac{\nu_{\mathfrak{a}}^{J}\left(p^{e}\right)}{p^{e}} .
$$

The set of such numbers for various $J$ form the jumping coefficients for the test ideals $\left\{\tau\left(\mathfrak{a}_{p}^{\alpha}\right)\right\}_{\alpha}$ introduced by Hara and Yoshida in [HY]. On the other hand, in characteristic zero we have the multiplier ideals of $\mathfrak{a}$, and the corresponding jumping coefficients (see [La] for the theory of multiplier ideals). We refer to $[\mathrm{MTW}]$ for an overview of results and open questions relating the jumping coefficients of the multiplier ideals and the $F$-thresholds for the reduction $\bmod p$, when $p \gg 0$.

Remark 2.5. As follows from the above discussion, the "top degree" part in $\nu_{\mathfrak{a}}^{J}\left(p^{e}\right)$ is related to the jumping coefficients of the multiplier ideals of $\mathfrak{a}$, while the "free term" is related to the roots of the Bernstein-Sato polynomial. Recall that there is also a direct connection between these roots and the jumping coefficients. More precisely, the largest root of $b_{\mathfrak{a}}$ is $-\operatorname{lc}(\mathfrak{a})$, where $\operatorname{lc}(\mathfrak{a})$ is the $\log$ canonical threshold of $\mathfrak{a}$ (this is the first nonzero jumping coefficient). Moreover, if $\lambda \in$ $[\operatorname{lc}(\mathfrak{a}), \operatorname{lc}(\mathfrak{a})+1)$ is a jumping coefficient, then $-\lambda$ is a root of $b_{\mathfrak{a}}$. These results are proved in $[\mathrm{Ko}]$ and [ELSV] in the codimension one case, and in [BMS2] in general.

We turn now to the monomial case, in which we will give an explicit description of the whole picture. As we will see, understanding the $F$-thresholds is quite easy. For example, this follows from the result in [HY] saying that for monomial ideals the test ideals are the same as the multiplier ideals. On the other hand, we will see that the invariants $\nu_{\mathfrak{a}}^{J}\left(p^{e}\right)$ give much more information. In particular, by the method described above we will be able to recover all the roots of the Bernstein-Sato polynomial.

\section{Bernstein-Sato polynomials of monomial ideals}

From now on we assume that $\mathfrak{a}$ is an ideal generated by monomials, so we may take $f_{j}=X^{a_{j}}=\prod_{i} X_{i}^{a_{i, j}}$ for all $j$, where $a_{j}=\left(a_{1, j}, \ldots, a_{n, j}\right) \in \mathbb{N}^{n}$. We consider the linear forms $\ell_{i}(s)=\sum_{j} a_{i, j} s_{j}$ on $\mathbb{Z}^{r}$.

In this section we give a direct proof of the existence of the Bernstein-Sato polynomial in this case. We first make use of the homogeneity of the ideal to reinterpret equation (1) as in [BMS2]. We consider, more generally, the ideal $I_{\mathfrak{a}} \subseteq \mathbb{C}\left[s_{1}, \ldots, s_{r}\right]$ consisting of those polynomials $F$ such that there is an equality of the form

$$
F\left(s_{1}, \ldots, s_{r}\right) f_{1}^{s_{1}} \ldots f_{r}^{s_{r}}=\sum_{c} P_{c} \cdot \prod_{j, c_{j}<0}\left(\begin{array}{c}
s_{j} \\
-c_{j}
\end{array}\right) f_{1}^{s_{1}+c_{1}} \ldots f_{r}^{s_{r}+c_{r}},
$$

with $c$ and $P_{c}$ as in (1). 
On $\mathbb{C}\left[X, X^{-1}, s\right]$ we consider the $\mathbb{Z}^{n}$-grading given by $\operatorname{deg}\left(s_{j}\right)=0$ and $\operatorname{deg}\left(X_{i}\right)=e_{i}$, the $i$ th element of the standard basis of $\mathbb{Z}^{n}$. This induces a grading on $\mathbb{C}\left[X, X^{-1}, s\right] \prod_{j} f_{j}^{s_{j}}$ such that $\operatorname{deg}\left(h \prod_{j} f_{j}^{s_{j}}\right)=\operatorname{deg}(h)$. On $\mathbb{C}\left[X, \partial_{X}, s\right]$ we have the corresponding grading with $\operatorname{deg}\left(\partial_{X_{i}}\right)=-\operatorname{deg}\left(X_{i}\right)$ and the action on $\mathbb{C}\left[X, X^{-1}, s\right] \prod_{j} f_{j}^{s_{j}}$ is compatible with the gradings.

Note that $\prod_{j} f_{j}^{s_{j}}=\prod_{i} X_{i}^{\ell_{i}(s)}$ and $\prod_{j} f_{j}^{s_{j}+c_{j}}=\prod_{i} X_{i}^{\ell_{i}(s+c)}$. It follows that in (4) we may assume that $\operatorname{deg}\left(P_{c}\right)=-\left(\ell_{1}(c), \ldots, \ell_{n}(c)\right)$ for all $c$. If $P \in$ $\mathbb{C}\left[X, \partial_{X}, s\right]$ has degree $m=\left(m_{i}\right)$, then

$$
P \in \mathbb{C}\left[X_{1} \partial_{X_{1}}, \ldots, X_{n} \partial_{X_{n}}, s\right] \cdot \prod_{i} \xi_{i}^{\left|m_{i}\right|},
$$

where $\xi_{i}=X_{i}$ if $m_{i} \geq 0$, and $\xi_{i}=\partial_{X_{i}}$ if $m_{i} \leq 0$.

For every $c \in \mathbb{Z}^{r}$, let

$$
g_{c}:=\prod_{j, c_{j}<0}\left(\begin{array}{c}
s_{j} \\
-c_{j}
\end{array}\right) \cdot \prod_{i, \ell_{i}(c)>0}\left(\begin{array}{c}
\ell_{i}(s)+\ell_{i}(c) \\
\ell_{i}(c)
\end{array}\right) .
$$

Proposition 3.1. With the above notation, we have

$$
I_{\mathfrak{a}}=\left(g_{c} \mid c \in \mathbb{Z}^{r}, \sum_{j} c_{j}=1\right)=\left(g_{c} \mid c \in \mathbb{Z}^{r}, \sum_{j} c_{j} \geq 1\right) .
$$

Proof. We get the first equality by the above argument, considering the action of $\partial_{X_{k}}^{\ell_{k}(c)}$ on $\prod_{i} X_{i}^{\ell_{i}(s+c)}$. For the second equality we need to show that if $c \in \mathbb{Z}^{r}$ and $\sum_{j} c_{j} \geq 1$, then $g_{c} \in I_{\mathfrak{a}}$. We get this by induction on $\sum_{j} c_{j}$. Indeed, if $\sum_{j} c_{j} \geq 2$ and $c_{j_{0}}>0$, let $c_{j}^{\prime}=c_{j}$ for $j \neq j_{0}$ and $c_{j_{0}}^{\prime}=c_{j_{0}}-1$. Since $\ell_{i}(c) \geq \ell_{i}\left(c^{\prime}\right)$, it follows that $g_{c^{\prime}}$ divides $g_{c}$. By the induction hypothesis, $g_{c^{\prime}}$ lies in $I_{\mathfrak{a}}$, hence so does $g_{c}$.

It follows from the above description of the generators of $I_{\mathfrak{a}}$ that for any irreducible component $\Gamma$ of $V\left(I_{\mathfrak{a}}\right)_{\text {red }}$, there are subsets $A \subseteq\{1, \ldots, r\}$ and $B \subseteq$ $\{1, \ldots, n\}$ together with $\alpha_{j}$ in $\mathbb{Z}_{\geq 0}$ for $j$ in $A$ and $\beta_{i}$ in $\mathbb{Z}_{<0}$ for $i$ in $B$ such that

$$
\Gamma=\left\{u=\left(u_{j}\right) \in \mathbb{C}^{r} \mid u_{j}=\alpha_{j} \text { for } j \text { in } A, \ell_{i}(u)=\beta_{i} \text { for } i \text { in } B\right\} .
$$

Moreover, after possibly enlarging $A$ and $B$, we may assume that if $j$ is not in $A$ and $u_{j}$ is constant on $\Gamma$, then $u_{j}$ is not in $\mathbb{Z}_{\geq 0}$ for $u$ in $\Gamma$, and if $i$ is not in $B$ and $\ell_{i}$ is constant on $\Gamma$, then $\ell_{i}(u)$ is not in $\mathbb{Z}_{<0}$ for $u$ in $\Gamma$.

Lemma 3.2. With the above notation and assumption, if $c$ in $\mathbb{Z}^{r}$ is such that $c_{j} \geq-\alpha_{j}$ for all $j$ in $A$ and $\ell_{i}(c) \leq-\beta_{i}-1$ for all $i$ in $B$, then $\sum_{j} c_{j} \leq 0$.

Proof. By Proposition 3.1, if $\sum_{j} c_{j} \geq 1$ then $\Gamma \subseteq g_{c}^{-1}(0)$. Therefore the assertion follows from the above maximality assumption.

Remark 3.3. We see that the converse of the above argument is also true: if $A, B$, the $\alpha_{j}$ and the $\beta_{i}$ are such that the assertion in Lemma 3.2 holds and if $\Gamma$ is given by $(5)$, then $\Gamma \subseteq V\left(I_{\mathfrak{a}}\right)_{\text {red }}$. 
By definition, the Bernstein-Sato polynomial of $\mathfrak{a}$ is the monic generator of $I_{\mathfrak{a}} \cap \mathbb{C}\left[s_{1}+\ldots+s_{r}\right]$. The existence of this polynomial follows from the next proposition.

Proposition 3.4. With the above notation, the intersection $I_{\mathfrak{a}} \cap \mathbb{C}\left[s_{1}+\ldots+s_{r}\right]$ is nonzero.

Proof. We need to show that for every irreducible component $\Gamma$ of $V\left(I_{\mathfrak{a}}\right)_{\text {red }}$, the map $u=\left(u_{j}\right) \in \Gamma \longrightarrow \sum_{j} u_{j}$ is constant. This is equivalent to the assertion that if $A$ and $B$ are such that $\Gamma$ is given by (5) and Lemma 3.2 holds, then for every $u$ in $\mathbb{C}^{r}$ such that $u_{j}=0$ for all $j$ in $A$ and $\ell_{i}(u)=0$ for all $i$ in $B$, we have $\sum_{j} u_{j}=0$. Since the vector space cut out by these equations is defined over $\mathbb{Q}$, we may assume that $u$ is in $\mathbb{Q}^{r}$ and moreover, that it is in $\mathbb{Z}^{r}$.

Suppose that $\sum_{j} u_{j}$ is nonzero. After possibly replacing $u$ by $-u$, we may assume that $\sum_{j} u_{j}>0$. In this case we get a contradiction by applying Lemma 3.2 for $c=u$. This completes the proof of the existence of the Bernstein-Sato polynomial $b_{\mathfrak{a}}$ in the monomial case.

Remark 3.5. It follows from the discussion before Proposition 3.1 that both $b_{\mathfrak{a}}$ and the $P_{c}$ satisfying (4) have rational coefficients. The above proof shows that all roots of $b_{\mathfrak{a}}$ are rational, since $\Gamma$ is an affine linear subspace defined by equations with rational coefficients. Moreover, we have obtained the following description of the roots: we need to consider all $A, B, \alpha_{j}$ and $\beta_{i}$ such that the assertion in Lemma 3.2 holds. If $\Gamma$ is defined by (5) and if it is nonempty, then $\sum_{j} u_{j}$ is constant on $\Gamma$, and its value gives a root of $b_{\mathfrak{a}}$. In addition, all the roots arise in this way.

\section{Roots of Bernstein-Sato polynomials for monomial ideals}

We study now the invariants from $\S 2$ in the case when $\mathfrak{a}$ is a monomial ideal. Proposition 6.1 in the Appendix shows that for every ideal $J \subseteq\left(X, \ldots, X_{n}\right) \subseteq$ $\mathbb{Z}\left[X_{1}, \ldots, X_{n}\right]$ such that $\mathfrak{a} \subseteq \operatorname{Rad}(J)$, there is a monomial ideal $\widetilde{J}$ such that if $p \gg 0$, then $\nu_{\mathfrak{a}}^{J}\left(p^{e}\right)=\nu_{\mathfrak{a}}^{J}\left(p^{e}\right)$ for every $e \geq 1$. Therefore in order to understand the functions $\nu_{\mathfrak{a}}^{J}$ for various $J$, it is enough to consider the case when $J$ is a monomial ideal, too. Our goal is to give an affirmative answer to Problem 2.3 in this setting and to show that all roots of $b_{\mathfrak{a}}$ are given by our method. Moreover, along the way we will get a description of these roots.

We assume that $\mathfrak{a}$ and $J$ are proper nonzero monomial ideals in $\mathbb{Z}\left[X_{1}, \ldots, X_{n}\right]$ such that $\mathfrak{a}$ is contained in the radical of $J$. Since we deal with monomial ideals, we can define the function $\nu_{\mathfrak{a}}^{J}$ without taking the reduction $\bmod p$. If $q$ is an arbitrary positive integer (not necessarily a prime power), we put

$$
J^{[q]}:=\left(X^{q w} \mid X^{w} \in J\right),
$$

where $X^{w}=\prod_{i} X_{i}^{w_{i}}$ for $w=\left(w_{1}, \ldots, w_{n}\right) \in \mathbb{N}^{n}$. We also define

$$
\nu_{\mathfrak{a}}^{J}(q):=\max \left\{t \geq 0 \mid \mathfrak{a}^{t} \nsubseteq J^{[q]}\right\} .
$$


Our first goal is to prove the following theorem. Note that it immediately gives a positive answer to Problem 2.3 for monomial ideals.

Theorem 4.1. If $\mathfrak{a}$ is a nonzero proper monomial ideal, then there is a positive integer $N$ with the following property. If $J$ is a monomial ideal whose radical contains $\mathfrak{a}$, then there are rational numbers $\alpha>0$ and $\gamma_{j}$ for $j=0, \ldots, N-1$, such that $\nu_{\mathfrak{a}}^{J}(q)=\alpha q+\gamma_{j}$ if $q \equiv j(\bmod N)$ and $q$ is large enough.

Remark 4.2. With the notation in the theorem, note that if $j$ and $N$ are relatively prime, then by Proposition 2.2 together with Dirichlet's Theorem, $b_{\mathfrak{a}}\left(\gamma_{j}\right) \equiv 0(\bmod p)$ for infinitely many primes $p$. Therefore $\gamma_{j}$ is a root of $b_{\mathfrak{a}}$ for every such $j$. Note also that if $p \gg 0$ is a prime, then $\alpha$ is equal to the $F$-pure threshold $\mathrm{c}^{J}\left(\mathfrak{a}_{p}\right)$.

The proof of Theorem 4.1 will give, in fact, a description of $\alpha$ and of the $\gamma_{j}$. Moreover, Theorem 4.9 below will show that all roots of $b_{\mathfrak{a}}$ are given by some $\gamma_{j}$ as above, for a suitable $J$ and some $j$ relatively prime to $N$.

We start with some preparations. Note first that we may write $J=\bigcap_{i=1}^{d} J_{i}$, where each $J_{i}$ is generated by powers of variables $X_{j}^{b_{j}}$ for $j \in I_{i} \subseteq\{1, \ldots, n\}$. This can be checked, for example, by induction on $n$. If Theorem 4.1 holds for every $J_{i}$, then it holds for $J$ : it is clear that we have $\nu_{\mathfrak{a}}^{J}(q)=\max _{i} \nu_{\mathfrak{a}}^{J_{i}}(q)$ for all $q$. The assertion follows from the fact that if a finite set $S$ is such that for every $i$ in $S$ the function $m \rightarrow h_{i}(m)$ is affine linear for $m$ large enough and in a suitable congruence class, then so is the function $m \longrightarrow \max _{i \in S} h_{i}(m)$. Therefore from now on we may assume that $J=\left(X_{i}^{b_{i}} \mid i \in I\right)$ for some $I \subseteq\{1, \ldots, n\}$.

Recall that we denote the generators of $\mathfrak{a}$ by $X^{a_{1}}, \ldots, X^{a_{r}}$, where $a_{j}=$ $\left(a_{1, j}, \ldots, a_{n, j}\right)$. We have $\mathfrak{a}^{t} \subseteq J^{[q]}$ if and only if for all $\beta=\left(\beta_{j}\right) \in \mathbb{N}^{r}$ with $\sum_{j} \beta_{j}=t$, there is $i$ in $I$ such that $q b_{i} \leq \sum_{j=1}^{r} a_{i, j} \beta_{j}$. Hence $\nu_{\mathfrak{a}}^{J}(q)=$ $\tau\left(I ;\left(q b_{i}-1\right)_{i \in I}\right)$, where for $w=\left(w_{i}\right) \in \mathbb{N}^{|I|}$, we put

$$
\tau(I ; w):=\max \left\{\sum_{j=1}^{r} \beta_{j} \mid \beta \in \mathbb{N}^{r}, \ell_{i}(\beta) \leq w_{i} \text { for } i \text { in } I\right\} .
$$

Recall that $\ell_{i}(\alpha)=\sum_{j=1}^{r} a_{i, j} \alpha_{j}$. We denote by $\ell_{i}$ also the extension of this linear function to $\mathbb{Q}^{r}$.

In order to simplify the notation we show that we may assume $I=\{1, \ldots, n\}$, after replacing, if necessary, $J$ by $J^{\prime}:=J+\left(X_{i}^{M} \mid i \notin I\right)$ for $M$ sufficiently large. Indeed, note first that if $b^{\prime}$ is in $\mathbb{N}^{|I|}$, then $\tau\left(I ; b^{\prime}\right)$ is finite, so the set

$$
\left\{\beta \in \mathbb{Q}_{+}^{r} \mid \ell_{i}(\beta) \leq b_{i}^{\prime} \text { for } i \text { in } I\right\}
$$

is bounded. This implies that if $M \gg 0$, then for every $q \geq 1$ and every $\beta \in \mathbb{N}^{n}$ such that $\ell_{i}(\beta)<q b_{i}$ for all $i$ in $I$, we have $\ell_{i}(\beta)<q M$ for all $i \notin I$. For such $M$ we have $\nu_{\mathfrak{a}}^{J}(q)=\nu_{\mathfrak{a}}^{J^{\prime}}(q)$ for every $q \geq 1$. 
From now on we assume that $I=\{1, \ldots, n\}$, and we put $\tau(w)$ for $\tau(I ; w)$. For every $w$ in $\mathbb{Q}_{+}^{n}$ we define also

$$
\tau_{\mathbb{Q}}(w):=\max \left\{\sum_{j=1}^{r} \alpha_{j} \mid \alpha \in \mathbb{Q}_{+}^{r}, \ell_{i}(\alpha) \leq w_{i} \text { for all } i\right\} .
$$

Here we may replace $\alpha \in \mathbb{Q}_{+}^{r}$ with $\alpha \in \mathbb{R}_{+}^{r}$ because $w$ is in $\mathbb{Q}_{+}^{n}$ and the $\ell_{i}$ have coefficients in $\mathbb{Q}$.

Let $P_{\mathfrak{a}}$ be the Newton polyhedron of $\mathfrak{a}$ and let $\Delta$ be the fan decomposition of $\mathbb{Q}_{+}^{n}$ whose cones are the closed convex cones over the faces of $P_{\mathfrak{a}}$. A maximal such cone corresponds to a facet $Q$ of $P_{\mathfrak{a}}$ that is not contained in a coordinate hyperplane, and we denote by $L_{Q}$ or by $L_{\sigma}$ the linear function such that $Q=$ $P_{\mathfrak{a}} \cap L_{Q}^{-1}(1)$.

Lemma 4.3. Let $P_{\mathfrak{a}}$ be the Newton polyhedron of $\mathfrak{a}$. If there is $\lambda>0$ such that $w$ is in $\lambda P_{\mathfrak{a}}$, then

$$
\tau_{\mathbb{Q}}(w)=\max \left\{\lambda>0 \mid w \in \lambda P_{\mathfrak{a}}\right\}
$$

and if there is no such $\lambda$, then $\tau_{\mathbb{Q}}(w)=0$. In particular, $\tau_{\mathbb{Q}}$ is piecewise linear on $\mathbb{Q}_{+}^{n}$ : if $\sigma$ is a maximal cone in $\Delta$, then $\tau_{\mathbb{Q}}=L_{\sigma}$ on $\sigma$. Moreover, there is a positive integer $N$ such that $\tau_{\mathbb{Q}}(w)=\tau(w)$ if $w_{i} / N \in \mathbb{N}$ for all $i$.

Proof. Since $P_{\mathfrak{a}}=\operatorname{conv}\left(a_{1}, \ldots, a_{r}\right)+\mathbb{R}_{+}^{n}$, where $\operatorname{conv}\left(a_{1}, \ldots, a_{r}\right)$ denotes the convex hull of the $a_{i}$, we see that $\frac{1}{\lambda} w$ is in $P_{\mathfrak{a}}$ if and only if there are $\beta_{1}, \ldots, \beta_{r} \geq$ 0 such that $\sum_{j} \beta_{j}=1$ and $w_{i} / \lambda \geq \ell_{i}(\beta)$ for all $i$, where $\beta=\left(\beta_{1}, \ldots, \beta_{r}\right)$. This gives the first assertion.

Let $w$ be in the closed cone $\sigma$ over a facet $Q$ of $P_{\mathfrak{a}}$. If there is $\lambda>0$ such that $w$ is in $\lambda P_{\mathfrak{a}}$, then for the largest such $\lambda$ we have $w$ in $\lambda Q$. Therefore $\lambda=L_{\sigma}(w)$. Since both $L_{\sigma}$ and $\tau_{\mathbb{Q}}$ are continuous, we deduce that $L_{\sigma}=\tau_{\mathbb{Q}}$ on $\sigma$.

We denote by $e_{1}, \ldots, e_{n}$ the standard basis of $\mathbb{R}^{n}$. Every maximal cone $\sigma$ in $\Delta$ is generated as a convex cone by some of the $a_{j}$ (the vertices of $Q$ ) and some of the vectors $e_{1}, \ldots, e_{n}$. By Carthéodory's theorem (see Proposition 1.15 in $[\mathrm{Zi}]) \sigma$ can be written as a union of cones, each generated by a subset of the generators of $\sigma$ that forms a basis of $\mathbb{R}^{n}$.

Suppose now that $w$ lies in the maximal cone $\sigma$. By the above discussion, we can write

$$
w=\sum_{j \in I_{1}} \alpha_{j} a_{j}+\sum_{i \in I_{2}} \beta_{i} e_{i} \quad \text { with } \alpha_{j} \text { and } \beta_{i} \text { in } \mathbb{R}_{+},
$$

where the $a_{j}$ and the $e_{i}$ lie in $\sigma$ and give a basis of $\mathbb{R}^{n}$. For the last assertion we need to find $N$ such that for every $w$ as above lying in $(N \mathbb{N})^{n}$, the $\alpha_{j}$ and the $\beta_{i}$ are integers.

Let $N$ be a positive number which is divisible by the determinant of any square submatrix of $\left(a_{i, j}\right)$. Using the determinant of the submatrix $\left(a_{i, j}\right)$, with $i$ in the complement of $I_{2}$ and $j$ in $I_{1}$, we see that $\alpha_{j}$ is an integer for every $j$ in $I_{1}$. This in turn implies that all $\beta_{i}$ are integers, and completes the proof of the lemma. 
With $N$ as in the above lemma, we have a good understanding of $\tau$ on $(N \mathbb{N})^{r}$. We describe now the behavior of $\tau$ on the congruence classes modulo the subgroup $(N \mathbb{Z})^{n}$. Let $\Delta$ be the fan decomposition in Lemma 4.3. Consider a cone $\sigma$ in $\Delta$ which is not contained in any of the coordinate hyperplanes, and a translate $v+\sigma$ of this cone, for some $v \in \mathbb{Z}^{n}$.

Lemma 4.4. With the above notation, there is $w$ in $\sigma \cap \mathbb{N}^{n}$ such that the restriction of $\tau$ to the intersection of each congruence class with $v+w+\sigma$ is given by an affine linear function. More precisely, for every $c=\left(c_{1}, \ldots, c_{n}\right)$ in $\{0, \ldots, N-1\}^{n}$ there is $A_{c}$ in $\mathbb{Q}_{+}$such that for every $u$ in $(v+w+\sigma) \cap \mathbb{N}^{n}$ with $u_{i} \equiv c_{i}(\bmod N)$ for all $i$, we have $\tau(u)=\tau_{\mathbb{Q}}(u)-A_{c}$.

Proof. Note that we may replace at any time $v$ by $v+v^{\prime}$ for some $v^{\prime}$ in $\sigma \cap \mathbb{N}^{n}$. If $\widetilde{\sigma}$ is a maximal cone in $\Delta$ such that $\sigma$ is a face of $\widetilde{\sigma}$, then by taking $v^{\prime}$ to be a large enough multiple of an element in the interior of $\widetilde{\sigma}$, we may assume that $v+\sigma$ is contained in $\widetilde{\sigma}$.

It is clear that $\tau$ is concave: if $b, b^{\prime} \in \mathbb{N}^{n}$ we have $\tau\left(b+b^{\prime}\right) \geq \tau(b)+\tau\left(b^{\prime}\right)$. On the other hand, $\tau(b)$ and $\tau_{\mathbb{Q}}(N b)=N \tau_{\mathbb{Q}}(b)$ are in $\mathbb{N}$, so

$$
0 \leq \tau_{\mathbb{Q}}(b)-\tau(b) \in \frac{1}{N} \mathbb{Z} .
$$

It follows that given $c$, we may choose $w_{c} \in \sigma \cap \mathbb{N}^{n}$ such that

$$
\begin{gathered}
\tau_{\mathbb{Q}}\left(v+w_{c}\right)-\tau\left(v+w_{c}\right)= \\
\min \left\{\tau_{\mathbb{Q}}(v+w)-\tau(v+w) \mid w \in \sigma \cap \mathbb{N}^{n}, v_{i}+w_{i} \equiv c_{i}(\bmod N) \text { for all } i\right\} .
\end{gathered}
$$

If $w^{\prime} \in \sigma \cap(N \mathbb{N})^{n}$, then by concavity we have

$$
\tau_{\mathbb{Q}}\left(v+w_{c}+w^{\prime}\right)-\tau\left(v+w_{c}+w^{\prime}\right) \leq \tau_{\mathbb{Q}}\left(v+w_{c}\right)-\tau\left(v+w_{c}\right)
$$

(note that $\tau_{\mathbb{Q}}$ is linear on $\widetilde{\sigma}$ ). By minimality, we have equality in (6). If we take $w=\sum_{c} w_{c}$, then we can find for every $c$ an $A_{c}$ as required by the lemma.

Remark 4.5. Note that if $\sigma$ is a maximal cone, then for every $c$ there are infinitely many $u$ in $(v+w+\sigma) \cap \mathbb{N}^{n}$ such that $u_{i} \equiv c_{i}(\bmod N)$ for every $i$. This is not necessarily the case if $\sigma$ is not maximal. However, if given $c$ there is one such $u$, then there are infinitely many with the same property.

We can solve now the monomial case of Problem 2.3.

Proof of Theorem 4.1. We use the notation in Lemmas 4.3 and 4.4. We have seen that we may assume $J=\left(X_{i}^{b_{i}} \mid 1 \leq i \leq n\right)$. Consider $N$ and the fan $\Delta$ in Lemma 4.3. Let $\sigma$ be the cone in $\Delta$ such that $b=\left(b_{i}\right)$ lies in the relative interior of $\sigma$ (note that since $b_{i}>0$ for every $i, \sigma$ is not contained in any coordinate hyperplane). We put $e=(1, \ldots, 1) \in \mathbb{N}^{n}$ and let $\widetilde{\sigma}$ be a maximal cone in $\Delta$ such that $q b-e \in \widetilde{\sigma}$ for $q \gg 0$. It follows that $\sigma$ is a face of $\widetilde{\sigma}$. Recall that we have a linear function $L_{\sigma}$ whose restriction to $\widetilde{\sigma}$ is equal to $\tau_{\mathbb{Q}}$.

Lemma 4.4 implies that we can find $v_{\sigma}$ in $\sigma \cap \mathbb{N}^{n}$ and for every $c \in\{0, \ldots, N-$ $1\}^{n}$, a nonnegative rational number $A_{c}^{\sigma}$ such that

$$
\tau_{\mathbb{Q}}(u)-\tau(u)=A_{c}^{\sigma}
$$


if $u$ is in $\left(v_{\sigma}-e+\sigma\right) \cap \mathbb{N}^{n}$ and $u_{i} \equiv c_{i}(\bmod N)$ for all $i$.

Recall that $\nu_{\mathfrak{a}}^{J}(q)=\tau(q b-e)$. Moreover, if $q$ is large enough, then $q b-e$ lies in $v_{\sigma}-e+\sigma$. Given $j \in\{0, \ldots, N-1\}$, we take $c=\left(c_{i}\right)$ such that $j b_{i}-1 \equiv c_{i}$ $(\bmod N)$ for all $i$. If we put $\alpha=L_{\sigma}(b)=\tau_{\mathbb{Q}}(b)$ and $\gamma_{j}=L_{\sigma}(-e)-A_{c}^{\sigma}$, then the requirement of the theorem is satisfied.

Remark 4.6. For future reference, we give explicitly the description of the roots of $b_{\mathfrak{a}}$ that are obtained by our method (Theorem 4.9 below shows that these are, indeed, all the roots of $b_{\mathfrak{a}}$ ). For every cone $\sigma$ in our fan $\Delta$, such that $\sigma$ is not contained in a coordinate hyperplane, let us choose a maximal cone $\widetilde{\sigma}$ in $\Delta$ with the property that for some $v$ in $\sigma$ we have $v-e+\sigma \subseteq \widetilde{\sigma}$ (hence $\sigma$ is a face of $\widetilde{\sigma})$. Let $L_{\sigma}$ be the linear function whose restriction to $\widetilde{\sigma}$ is equal to $\tau_{\mathbb{Q}}$.

We consider now those $c$ in $\{0, \ldots, N-1\}^{n}$ such that there is $b$ in $\mathbb{N}^{n}$ in the relative interior of $\sigma$ with $b_{i}-1 \equiv c_{i}(\bmod N)$ for all $i$ (if $\sigma$ is maximal, then all $c$ satisfy this condition). With $A_{c}^{\sigma}$ as in the proof of Theorem 4.1, we deduce from Remark 4.2 that $L_{\sigma}(-e)-A_{c}^{\sigma}$ is a root of $b_{\mathfrak{a}}$. Indeed, it is enough to consider $j$ with $j \equiv 1(\bmod N)$ and $J=\left(X_{1}^{b_{1}}, \ldots, X_{n}^{b_{n}}\right)$. In addition, every root we obtain by our method is of this form. Note that the roots we obtain for $\sigma \in \Delta$ do not depend on the choice of $\widetilde{\sigma}$ : if $\widetilde{\sigma}^{\prime}$ is another maximal cone that satisfies the same property, then $L_{\sigma}$ and $L_{\sigma^{\prime}}$ agree on the linear span of $\sigma$ and $e$.

Remark 4.7. With the notation in the previous remark, the class of the root $L_{\sigma}(-e)-A_{c}^{\sigma}$ in $\mathbb{Q} / \mathbb{Z}$ is equal to the class of $L_{\sigma}(-e)-L_{\sigma}(q w-e)=-q L_{\sigma}(w)$, where $w \in \mathbb{N}^{n}$ is in the relative interior of $\sigma$ and $q \gg 0$ are such that $w_{i}-1 \equiv c_{i}$ $(\bmod N)$ for all $i$ and $q \equiv 1(\bmod N)$. Recall that $N L_{\sigma}(w)$ is an integer for all such $w$. We see that in order to compute the set of all such classes, when $\sigma$ and $c$ vary, it is enough to consider only the maximal cones $\sigma$. The set we get in this way is the set of classes of

$$
\left\{-\tau_{\mathbb{Q}}(w) \mid w \in\left(\mathbb{Z}_{>0}\right)^{n}\right\} .
$$

Remark 4.8. It follows from Remark 4.6 that we get the same roots of the Bernstein-Sato polynomial if we consider only the invariants $\nu_{\mathfrak{a}}^{J}(p)$ for ideals $J$ of the form $\left(X_{1}^{b_{1}}, \ldots, X_{n}^{b_{n}}\right)$, and for $p$ prime and large enough with $p \equiv 1(\bmod$ $N)$.

The following theorem shows that all the roots of the Bernstein-Sato polynomial of a monomial ideal are detected by our method.

Theorem 4.9. For every nonzero monomial ideal $\mathfrak{a}$, and for every root $\lambda$ of $b_{\mathfrak{a}}$ there is a monomial ideal $J$ together with a rational number $\alpha$ and a positive integer $N^{\prime}$ such that $\nu_{\mathfrak{a}}^{J}(q)=\alpha q+\lambda$ for $q$ sufficiently large and with $q \equiv 1$ (mod $\left.N^{\prime}\right)$.

Corollary 4.10. The procedure described in Remark 4.6 gives all the roots of the Bernstein-Sato polynomial $b_{\mathfrak{a}}$. 
Proof. If $\lambda$ is a root of $b_{\mathfrak{a}}$, let $J, \alpha$ and $N^{\prime}$ be as in Theorem 4.9. Applying Theorem 4.1 for $\mathfrak{a}$ and $J$, we see that there are $\alpha^{\prime}$ and $\lambda^{\prime}$ such that $\nu_{\mathfrak{a}}^{J}(q)=$ $\alpha^{\prime} q+\lambda^{\prime}$ if $q$ is large enough and $q \equiv 1(\bmod N)$. Taking $q \equiv 1\left(\bmod N N^{\prime}\right)$, we deduce $\alpha^{\prime}=\alpha$ and $\lambda^{\prime}=\lambda$, which shows that $\lambda$ is obtained by the procedure described in Remark 4.6.

Proof of Theorem 4.9. We use the description of the roots of $b_{\mathfrak{a}}$ from Remark 3.5. We can find $A \subseteq\{1, \ldots, r\}, B \subseteq\{1, \ldots, n\}$ and $\left(\alpha_{j}\right)_{j \in A}$ in $\mathbb{Z}_{\geq 0}^{|A|}$ and $\left(\beta_{i}\right)_{i \in B}$ in $\mathbb{Z}_{<0}^{|B|}$ such that

$$
\Gamma:=\left\{w=\left(w_{j}\right) \in \mathbb{Q}^{r} \mid w_{j}=\alpha_{j} \text { for } j \text { in } A \text { and } \ell_{i}(w)=\beta_{i} \text { for } i \text { in } B\right\}
$$

is nonempty, and if $w \in \Gamma$, then $\lambda=\sum_{j=1}^{r} w_{j}$. Moreover, we may assume by Lemma 3.2 that the following condition holds: if $u=\left(u_{j}\right)$ in $\mathbb{Z}^{r}$ is such that $u_{j} \geq-\alpha_{j}$ for all $j$ in $A$ and $\ell_{i}(u) \leq-\beta_{i}-1$ for all $i$ in $B$, then $\sum_{j=1}^{r} u_{j} \leq 0$.

Let us fix $w$ in $\Gamma$. We deduce from our condition on $\left(\alpha_{j}\right)$ and $\left(\beta_{i}\right)$ that

$$
\lambda=\max _{v} \sum_{j=1}^{r} v_{j},
$$

the maximum being over those $v$ in $\mathbb{Q}^{r}$ such that $v_{j} \geq 0$ for $j$ in $A, \ell_{i}(v) \leq-1$ for $i$ in $B$ and $v_{j}-w_{j} \in \mathbb{Z}$ for all $j$ (the maximum is achieved for $v=w$ ).

We choose now $u=\left(u_{j}\right)$ in $\mathbb{Q}_{+}^{r}$ such that $u_{j}=0$ if and only if $j$ is in $A$, and such that $u+w$ is in $\mathbb{Z}^{r}$. Moreover, we may choose $u$ such that $\ell_{i}(u)=$ $\sum_{j=1}^{r} a_{i, j} u_{j}>0$ for all $i$ in $B$. Indeed, if this is not the case, then there is $i$ in $B$ such that $a_{i, j}=0$ whenever $j$ is not in $A$. This contradicts the fact that $\ell_{i}(w)<0$. Note also that since $u+w$ lies in $\mathbb{Z}^{r}, \ell_{i}(u)$ is an integer for every $i$ in $B$.

Let $N^{\prime}$ be a positive integer such that $N^{\prime} u$ is in $\mathbb{Z}_{\geq 0}^{r}$. If $q=m N^{\prime}+1$ for $m \geq 1$, then $q u+w$ is in $\mathbb{Z}^{r}$. With the notation introduced for the proof of Theorem 4.1, we claim that if $q$ as above is large enough, then we have

$$
\tau\left(B ;\left(q \ell_{i}(u)-1\right)_{i \in B}\right)=q \sum_{j=1}^{r} u_{j}+\lambda .
$$

This implies the assertion of the theorem: take $J=\left(X_{i}^{\ell_{i}(u)} \mid i \in B\right)$ and $\alpha=$ $\sum_{j=1}^{r} u_{j}$.

In order to prove the claim, suppose that $v \in \mathbb{Z}_{\geq 0}^{r}$ is such that $\ell_{i}(v) \leq$ $q \ell_{i}(u)-1$ for all $i$ in $B$. For $j$ in $A$ we have $v_{j}-q u_{j}=v_{j} \geq 0$, and for $i$ in $B$ we have $\ell_{i}(v-q u) \leq-1$. As all $v_{j}-q u_{j}-w_{j}$ are integers, we deduce from (7) that $\sum_{j=1}^{r} v_{j} \leq q \sum_{j=1}^{r} u_{j}+\lambda$.

On the other hand, if $q \gg 0$ then $q u_{j}+w_{j} \geq 0$ for all $j$. Note that $\ell_{i}(q u+w) \leq$ $q \ell_{i}(u)-1$ for $i$ in $B$ and $\sum_{j=1}^{r}\left(q u_{j}+w_{j}\right)=q \sum_{j} u_{j}+\lambda$. This completes the proof of the claim, and hence that of the theorem.

We can prove now the description of the classes $\bmod \mathbb{Z}$ of the roots of the Bernstein-Sato polynomial. 
Proof of Corollary 1.1. We use Theorems 4.1 and 4.9. Recall that we have seen in Remark 4.7 that the classes in $\mathbb{Q} / \mathbb{Z}$ of the roots of $b_{\mathfrak{a}}$ are equal to

$$
\left\{-L_{\sigma}(w)+\mathbb{Z} \mid \sigma \text { maximal cone in } \Delta, w \in \sigma \cap\left(\mathbb{Z}_{>0}\right)^{n}\right\} .
$$

Therefore in order to prove the theorem it is enough to show that for every maximal cone $\sigma$ in $\Delta$, the set of classes $\left\{-L_{\sigma}(w)+\mathbb{Z} \mid w \in \sigma \cap\left(\mathbb{Z}_{>0}\right)^{n}\right\}$ is the subgroup of $\mathbb{Q} / \mathbb{Z}$ generated by $\frac{1}{m_{\sigma}}$, where if $Q$ is the facet of $P_{\mathfrak{a}}$ corresponding to $\sigma$, we put $m_{\sigma}$ for $m_{Q}$.

Since $m_{\sigma} L_{\sigma}$ has integer coefficients, for every $w$ in $\sigma \cap\left(\mathbb{Z}_{>0}\right)^{n}$ the class of $-L_{\sigma}(w)$ lies in the subgroup generated by $1 / m_{\sigma}$. On the other hand, if $\tau \subseteq \sigma$ is a convex cone generated by a basis $e_{1}^{\prime}, \ldots, e_{n}^{\prime}$ for $\mathbb{Z}^{n}$, then $m_{\sigma}$ is the smallest positive integer such that all $m_{\sigma} L_{\sigma}\left(e_{i}^{\prime}\right)$ are integers. In this case it follows easily that there is $w$ in the interior of $\tau$ such that $\frac{1}{m_{\sigma}}+L_{\sigma}(w)$ is an integer. By taking suitable multiples of $w$ we see that the subgroup generated by the class of $\frac{1}{m_{\sigma}}$ is contained in (9), which completes the proof.

Example 4.11. As we have already mentioned, it is a general fact that the largest root of the Bernstein-Sato polynomial of $\mathfrak{a}$ is $-\operatorname{lc}(\mathfrak{a})$, where $\operatorname{lc}(\mathfrak{a})$ is the $\log$ canonical threshold of $\mathfrak{a}$ (see $[\mathrm{BMS} 2])$. Let us prove this for monomial ideals using the above results.

It follows from [Ho1] that $\mathrm{lc}(\mathfrak{a})$ is the largest positive real number $c$ such that $e=(1, \ldots, 1)$ lies in $c P_{\mathfrak{a}}$, where $P_{\mathfrak{a}}$ is the Newton polyhedron of $\mathfrak{a}$. Lemma 4.3 implies that, with our notation, $\operatorname{lc}(\mathfrak{a})=\tau_{\mathbb{Q}}(e)$.

Let $\sigma_{0}$ be a maximal cone in the fan $\Delta$ such that $e$ lies in $\sigma_{0}$. With the notation in Remark 4.6, we get the root $-\operatorname{lc}(\mathfrak{a})$ as $L_{\sigma_{0}}(-e)-A_{0}^{\sigma_{0}}$ (corresponding to $c=(0, \ldots, 0))$.

On the other hand, if $\lambda$ is another root of $b_{\mathfrak{a}}$, then by Theorem 4.9 and Remark 4.6 we have $\lambda=L_{\sigma}(-e)-A_{c}^{\sigma}$ for some $\sigma, \widetilde{\sigma}$ and some $c$. Since $A_{c}^{\sigma} \geq 0$ and since concavity of $\tau_{\mathbb{Q}}$ gives $L_{\sigma}(e) \geq \tau_{\mathbb{Q}}(e)=L_{\sigma_{0}}(e)$, we get $\lambda \leq-\operatorname{lc}(\mathfrak{a})$.

We end this section with a description of the $F$-thresholds of monomial ideals (see $\S 2$ for the definition). It follows from Proposition 6.1 in the Appendix that the set of all $F$-thresholds of the monomial ideal $\mathfrak{a}$ (computed for the reduction $\bmod p$ of $\mathfrak{a}$, where $p \gg 0$ is a prime) is equal to the set of $F$-thresholds $c^{J}\left(\mathfrak{a}_{p}\right)$ with respect to monomial ideals $J$. Moreover, we have seen that it is enough to consider the case when $J=\left(X_{1}^{b_{1}}, \ldots, X_{n}^{b_{n}}\right)$, where $b_{i}$ are positive integers. With the notation in the proof of Theorem 4.1, we have shown that for this $J$ we have

$$
\mathrm{c}^{J}\left(\mathfrak{a}_{p}\right)=\tau_{\mathbb{Q}}(b)
$$

for every prime $p$ large enough. We will denote this number simply by $c^{J}(\mathfrak{a})$, as it does not depend on $p$.

We make the connection with the multiplier ideals of $\mathfrak{a}$. Recall that by the description in [Ho1], the multiplier ideal $\mathcal{I}\left(\mathfrak{a}^{\alpha}\right)$ of $\mathfrak{a}$ with exponent $\alpha$ can be 
described as follows

$$
\mathcal{I}\left(\mathfrak{a}^{\alpha}\right)=\left(X^{w} \mid u+e \in \operatorname{Int}\left(\alpha P_{\mathfrak{a}}\right)\right) .
$$

The jumping coefficients of the multiplier ideals defined in [ELSV] are those $\alpha>0$ such that $\mathcal{I}\left(\mathfrak{a}^{\alpha}\right)$ is strictly contained in $\mathcal{I}\left(\mathfrak{a}^{\alpha-\epsilon}\right)$ for every $\epsilon>0$. It follows from the above description of the multiplier ideals of $\mathfrak{a}$, that every $b=\left(b_{i}\right)$ with $b_{i}$ positive integers gives a jumping coefficient $\alpha$ characterized by the fact that $b$ lies in the boundary of $\alpha P_{\mathfrak{a}}$. Moreover, every jumping coefficient arises in this way. The connection with the $F$-thresholds is given by

Proposition 4.12. For every prime $p$, the jumping coefficient corresponding to $b=\left(b_{i}\right)$ as above is equal to $\mathrm{c}^{J}\left(\mathfrak{a}_{p}\right)$, where $J=\left(X_{1}^{b_{1}}, \ldots, X_{n}^{b_{n}}\right)$.

Proof. The assertion follows from Lemma 4.3.

Remark 4.13. It follows from the above discussion that the $F$-thresholds in the monomial case are easy to describe. Alternatively, this can be seen as follows: it is shown in [MTW] that in general, the $F$-thresholds (for various $J$ ) are the jumping coefficients for the test ideals introduced in $[\mathrm{HY}]$ as an analogue of multiplier ideals. On the other hand, it is proved in [HY] that for monomial ideals the test ideals coincide with the multiplier ideals. This gives a different approach to the description in Proposition 4.12.

Remark 4.14. It is clear that for monomial ideals all $F$-thresholds are rational numbers. In fact, more is true. If $p$ is a prime, then the series $\sum_{e} \nu_{\mathfrak{a}}^{J}\left(p^{e}\right) t^{e}$ is rational. This follows from the fact that the function $e \rightarrow \nu_{\mathfrak{a}}^{J}\left(p^{e+1}\right)-p \nu_{\mathfrak{a}}^{J}\left(p^{e}\right)$ is eventually periodic, which is a consequence of Theorem 4.1 .

\section{Examples}

In this section we give some examples to illustrate how to use our approach to give roots of the Bernstein-Sato polynomial. We use freely the notation introduced for the proof of Theorem 4.1. In all these examples we do not describe the complete picture given in Remark 4.6, but we give enough information to recover all the roots. For more complicated examples, based on a more explicit combinatorial description of the roots of the Bernstein-Sato polynomial, we refer to [BMS1].

Example 5.1. Let $\mathfrak{a}=\left(\prod_{j \neq i} X_{j} \mid 1 \leq i \leq 4\right)$ in $\mathbb{Z}\left[X_{1}, \ldots, X_{4}\right]$, so we have $\ell_{i}(s)=\sum_{j \neq i} s_{j}$ for $1 \leq i \leq 4$. If $\alpha \in \mathbb{N}^{4}$ is such that $\ell_{i}(\alpha) \leq b_{i}$ for all $i$, summing these inequalities we get

$$
\sum_{i} \alpha_{i} \leq\left\lfloor\left(\sum_{i} b_{i}\right) / 3\right\rfloor
$$

where we use the notation $\lfloor x\rfloor$ for the largest integer $\leq x$.

Consider first the cone

$$
\sigma=\left\{w \mid w_{i} \geq 0, \sum_{j} w_{j} \geq 3 w_{i} \text { for all } i\right\}
$$


If $b \in \sigma \cap \mathbb{N}^{4}$, then $\tau(b)=\left\lfloor\left(\sum_{i} b_{i}\right) / 3\right\rfloor$. Indeed, suppose for example that $\sum_{i} b_{i} \equiv 2(\bmod 3)$. If we take $\alpha_{i}=\left(\sum_{j} b_{j}-2\right) / 3-\left(b_{i}-1\right)$ for $i=1,2$ and $\alpha_{i}=\left(\sum_{j} b_{j}-2\right) / 3-b_{i}$ for $i=3,4$, then $\alpha \in \mathbb{N}^{4}$ and $\sum_{i} \alpha_{i}=\left(\sum_{i} b_{i}-2\right) / 3$. The other two cases are similar. We get $L_{i}$ for $i=0,1,2$ with $L_{i}(b)=\left(\sum_{j} b_{j}-i\right) / 3$, such that whenever $b \in \sigma \cap \mathbb{N}^{4}$, with $\sum_{i} b_{i} \equiv i(\bmod 3)$ we have $\tau(b)=L_{i}(b)$. All $L_{i}(-1, \ldots,-1)$ are roots of $b_{\mathfrak{a}}$, which gives the roots $-\frac{4}{3},-\frac{5}{3}$ and -2 .

Suppose now that $b \in \mathbb{N}^{4}$ is such that, for example, $2 b_{4}>b_{1}+b_{2}+b_{3}$. If $\ell_{i}(\alpha) \leq b_{i}$ for all $i$, adding these inequalities for $1 \leq i \leq 3$ implies

$$
\sum_{i=1}^{4} \alpha_{i} \leq\left\lfloor\left(\sum_{i=1}^{3} b_{i}\right) / 2\right\rfloor .
$$

If we assume, in addition, that $b_{1}+b_{2} \geq b_{3}, b_{1}+b_{3} \geq b_{2}$ and $b_{2}+b_{3} \geq b_{1}$, then this maximum can be achieved. Indeed, if $\sum_{i=1}^{3} b_{i}$ is even, take $\alpha_{j}=\left(\sum_{i=1}^{3} b_{i}\right) / 2-b_{j}$ for $1 \leq j \leq 3$ and $\alpha_{4}=0$. If $\sum_{i=1}^{3} b_{i}$ is odd, take $\alpha_{1}=\left(\sum_{i=1}^{3} b_{i}-1\right) / 2-\left(b_{1}-1\right)$, $\alpha_{j}=\left(\sum_{i=1}^{3} b_{i}-1\right) / 2-b_{j}$ for $j=2,3$, and $\alpha_{4}=0$.

If we take $L_{i}^{\prime}$ for $i=0,1$ given by $L_{i}^{\prime}(b)=\left(\sum_{j=1}^{3} b_{j}-i\right) / 2$, we see that if $b$ is as above and $\sum_{j=1}^{3} b_{j} \equiv i(\bmod 2)$, we have $\tau(b)=L_{i}^{\prime}(b)$. Therefore both $L_{i}^{\prime}(-1, \ldots,-1)$ are roots of $b_{\mathfrak{a}}$, which gives the roots $-\frac{3}{2}$ and -2 .

Therefore we have obtained the roots $-\frac{3}{2},-\frac{4}{3},-\frac{5}{3}$ and -2 . Note that, in fact, by [BMS2] $(4.5) b_{\mathfrak{a}}=\left(s+\frac{3}{2}\right)\left(s+\frac{4}{3}\right)\left(s+\frac{5}{3}\right)(s+2)^{3}$.

Example 5.2. Let $\mathfrak{a}=\left(X^{2} Y Z, X Y^{2} Z, X Y Z^{2}\right)$, so $\ell_{1}(s)=2 s_{1}+s_{2}+s_{3}, \ell_{2}(s)=$ $s_{1}+2 s_{2}+s_{3}$ and $\ell_{3}(s)=s_{1}+s_{2}+2 s_{3}$.

If $\alpha \in \mathbb{N}^{3}$ is such that $\ell_{i}(\alpha) \leq b_{i}$, by summing these relations we get $\sum_{i} \alpha_{i} \leq$ $\left\lfloor\frac{1}{4} \sum_{i} b_{i}\right\rfloor$. This maximum is achieved if $b$ lies in the cone

$$
\sigma=\left\{w \mid w_{i} \geq 0, w_{i} \geq \frac{\sum_{j} w_{j}}{4} \text { for all } i\right\}
$$

Indeed, suppose for example that $\sum_{j} b_{j} \equiv 1(\bmod 4)$. If

$$
\alpha_{1}=b_{1}-\frac{\sum_{i} b_{i}-1}{4}, \alpha_{2}=b_{2}-\frac{\sum_{i} b_{i}-1}{4}, \text { and } \alpha_{3}=b_{3}-\frac{\sum_{i} b_{i}+3}{4},
$$

then $\alpha \in \mathbb{N}^{3}$ and $\sum_{i} \alpha_{i}=\left(b_{1}+b_{2}+b_{3}-1\right) / 4$. The other three cases are similar.

We get $L_{i}(b)=\left(\sum_{j} b_{j}-i\right) / 4$ for $i=0,1,2,3$ such that if $b \in \mathbb{N}^{3}$ is in $\sigma$ and $\sum_{j} b_{j} \equiv i(\bmod 4)$, then $\tau(b)=L_{i}(b)$. We get roots of the Bernstein-Sato polynomial of $\mathfrak{a}$ given by $L_{i}(-1,-1,-1)$ for $i=0,1,2,3$, i.e. $-\frac{3}{4},-1,-\frac{5}{4}$ and $-\frac{6}{4}$. Note that by $[\mathrm{BMS} 2](4.5), b_{\mathfrak{a}}(s)=\left(s+\frac{3}{4}\right)\left(s+\frac{5}{4}\right)\left(s+\frac{6}{4}\right)(s+1)^{3}$.

Example 5.3. Let $\mathfrak{a}$ be the ideal generated by $X_{i} X_{j}$ for all $1 \leq i<j \leq n$, with $n \geq 3$. We have

$$
\ell_{i}(s)=\sum_{j<i} s_{j, i}+\sum_{j>i} s_{i, j}
$$


for $s=\left(s_{i, j}\right)_{i<j}$. Note that if $\ell_{i}(s) \leq b_{i}$ for all $i$, by taking the sum we get

$$
\sum_{i<j} s_{i, j} \leq\left\lfloor\left(\sum_{i} b_{i}\right) / 2\right\rfloor \text {. }
$$

It is easy to give, as above, a full-dimensional cone $\sigma$ on which this maximum is achieved. This gives $L_{1}$ and $L_{2}$ with $L_{i}(b)=\left(\sum_{j} b_{j}-i\right) / 2$ such that if $b \in \mathbb{N}^{n}$ lies in $\sigma$ and $\sum_{j} b_{j} \equiv i(\bmod 2)$, then $\tau_{i}(b)=L_{i}(b)$. Therefore $L_{0}(-1, \ldots,-1)=-\frac{n}{2}$ and $L_{1}(-1, \ldots,-1)=-\frac{n+1}{2}$ are roots of $b_{\mathfrak{a}}$.

Suppose now that $b \in \mathbb{N}^{n}$ is such that $b_{1}>\sum_{j=2}^{n} b_{j}$. If $\ell_{i}(\alpha) \leq b_{i}$ for all $i$, by adding these inequalities for $i \geq 2$, we deduce $\sum_{i<j} \alpha_{i, j} \leq \sum_{j \geq 2} b_{j}$. Moreover, this maximum can be achieved: let $\alpha_{1, i}=b_{i}$ for $i>1$ and all other $\alpha_{i, j}=0$. If $L(b)=\sum_{j \geq 2} b_{j}$ we see that if $b$ is as above, then $\tau(b)=L(b)$. Hence $L(-1, \ldots,-1)=-(n-1)$ is also a root of $b_{\mathbf{a}}$. Note that by [BMS2] (4.5), the Bernstein-Sato polynomial of $\mathfrak{a}$ is $b_{\mathfrak{a}}(s)=\left(s+\frac{n}{2}\right)\left(s+\frac{n+1}{2}\right)(s+n-1)$.

\section{Appendix}

We show that if $\mathfrak{a}$ is a monomial ideal, then in order to compute the functions $\nu_{\mathfrak{a}}^{J}\left(p^{e}\right)$ for $p \gg 0$, it is enough to consider the case when $J$ is a monomial ideal.

Proposition 6.1. Let $\mathfrak{a}$ be a nonzero ideal generated by monomials in $\mathbb{Z}\left[X_{1}, \ldots, X_{n}\right]$ and let $J \subseteq\left(X_{1}, \ldots, X_{n}\right)$ be an ideal such that $\mathfrak{a}$ is contained in the radical of $J$. If we put

$$
\widetilde{J}:=\left(X^{u} \mid h X^{u} \in J \text { for some } h \in \mathbb{Z}\left[X_{1}, \ldots, X_{n}\right] \backslash\left(X_{1}, \ldots, X_{n}\right)\right)
$$

and if $p \gg 0$, then $\nu_{\mathfrak{a}}^{J}\left(p^{e}\right)=\nu_{\mathfrak{a}}^{J}\left(p^{e}\right)$ for every $e \geq 1$.

For the proof of Proposition 6.1 we will need the following lemma.

Lemma 6.2. With the notation in Proposition 6.1, for $p \gg 0$ we have

$$
\widetilde{J}_{p}=\left(X^{u} \mid X^{u} \in J_{p}\right)
$$

as ideals in $\mathbb{F}_{p}\left[X_{1}, \ldots, X_{n}\right]_{\left(X_{1}, \ldots, X_{n}\right)}$.

Proof. Given $X^{u}$ in $\widetilde{J}$ and $h \in \mathbb{Z}\left[X_{1}, \ldots, X_{n}\right]$ such that $h(0) \neq 0$ and $h X^{u} \in J$, then for $p$ not dividing $h(0)$ we see that the image of $X^{u}$ in $\mathbb{F}_{p}\left[X_{1}, \ldots, X_{n}\right]_{\left(X_{1}, \ldots, X_{n}\right)}$ lies in $J_{p}$. Since $\widetilde{J}$ is finitely generated, for $p \gg 0$ we deduce the inclusion $\subseteq$ in the statement.

We prove now the reverse inclusion. It is clear that if $a=\left(a_{i}\right)$ and $b=\left(b_{i}\right)$ in $\mathbb{N}^{n}$ are such that $a_{i} \leq b_{i}$ for every $i$, then $\left(J: X^{a}\right) \subseteq\left(J: X^{b}\right)$. For a subset $A$ of $\{1, \ldots, n\}$ and $w=\left(w_{i}\right) \in \mathbb{N}^{n}$, we denote by $w+\mathbb{N}^{A}$ the set

$$
\left\{u=\left(u_{i}\right) \in \mathbb{N}^{n} \mid u_{i} \geq w_{i} \text { for } i \in A \text { and } u_{i}=w_{i} \text { for } i \notin A\right\} .
$$

Using the fact that $\mathbb{Z}\left[X_{1}, \ldots, X_{n}\right]$ is Noetherian, we deduce that there is a decomposition

$$
\mathbb{N}^{n}=\bigsqcup_{j=1}^{m}\left(w^{(j)}+\mathbb{N}^{A_{j}}\right),
$$


for some $w^{(j)}$ in $\mathbb{N}^{n}$ and $A_{j} \subseteq\{1, \ldots, n\}$ such that for every $w$ in $w^{(j)}+\mathbb{N}^{A_{j}}$ the ideal $\left(J: X^{w}\right)$ is equal to a fixed ideal $I_{j}$. In particular, we deduce that for every $i$ in $A_{j}$, we have $\left(I_{j}: X_{i}\right)=I_{j}$.

Given a prime $p$, for every ideal $I$ in $\mathbb{Z}\left[X_{1}, \ldots, X_{n}\right]$ we denote by $\bar{I}$ the reduction of $I$ in $\mathbb{F}_{p}\left[X_{1}, \ldots, X_{n}\right]$. There is $p_{0}$ such that for every prime $p \geq p_{0}$ the following hold:

$$
\begin{gathered}
\left(\overline{I_{j}}: X_{i}\right)=\overline{I_{j}} \text { in } \mathbb{F}_{p}\left[X_{1}, \ldots, X_{n}\right] \text { for all } j \leq m \text { and all } i \in A_{j}, \\
\left(\bar{J}: X^{w^{(j)}}\right)=\overline{I_{j}} \text { in } \mathbb{F}_{p}\left[X_{1}, \ldots, X_{n}\right] \text { for all } j \leq m .
\end{gathered}
$$

In order to show (10), use the exact sequence

$$
0 \rightarrow\left(I_{j}: X_{i}\right) / I_{j} \rightarrow \mathbb{Z}\left[X_{1}, \ldots, X_{n}\right] / I_{j} \stackrel{\phi}{\rightarrow} \mathbb{Z}\left[X_{1}, \ldots, X_{n}\right] / I_{j},
$$

where $\phi$ is multiplication by $X_{i}$. Note that given an arbitrary finitely generated $\mathbb{Z}\left[X_{1}, \ldots, X_{n}\right]$-module $M$, there is a positive integer $\ell$ such that $M[1 / \ell]$ is flat over $\mathbb{Z}[1 / \ell]$. Indeed, the submodule $N \subseteq M$ of elements annihilated by some positive integer is finitely generated over $\mathbb{Z}\left[X_{1}, \ldots, X_{n}\right]$. Therefore we can find $\ell$ such that $\ell N=0$, and $M[1 / \ell]$ is torsion-free, hence flat over $\mathbb{Z}[1 / \ell]$. Applying this observation for the image and for the cokernel of $h$, we see that if $p \gg 0$, then the sequence obtained from (12) by tensoring with $\mathbb{F}_{p}$ is again exact, which gives (10). The proof of (11) is similar.

Consider now a prime $p \geq p_{0}$ and fix $w \in \mathbb{N}^{n}$. Let $j$ be such that $w$ is in $w^{(j)}+\mathbb{N}^{A_{j}}$. Suppose that $h$ is in $\mathbb{F}_{p}\left[X_{1}, \ldots, X_{n}\right]$ such that $h(0) \neq 0$ and $h X^{w}$ is in $\bar{J}$. Using (11) we deduce that $h X^{w-w^{(j)}}$ lies in $\overline{I_{j}}$. Moreover, (10) implies that $h$ is in $\overline{I_{j}}$. If $g$ is an element in $I_{j}$ whose class is $h$, then $g(0) \neq 0$ and $g X^{w^{(j)}}$ is in $J$. In particular, $g X^{w}$ is in $J$, so $X^{w} \in \widetilde{J}$, which completes the proof of the lemma.

Proof of Proposition 6.1. We consider $p \gg 0$ so the assertion in Lemma 6.2 applies. In particular, we have $\widetilde{J}_{p} \subseteq J_{p}$, so $\nu_{\mathfrak{a}}^{J}\left(p^{e}\right) \geq \nu_{\mathfrak{a}}^{J}\left(p^{e}\right)$. In order to show that we have equality, since $\mathfrak{a}$ is monomial it is enough to prove that if $X^{u}$ is in $J_{p}^{\left[p^{e}\right]}$, then $X^{u}$ is in $\widetilde{J}_{p}^{\left[p^{e}\right]}$. We do induction on $e \geq 0$, the case $e=0$ being a consequence of Lemma 6.2.

If $e \geq 1$, we write $u=p v+w$ with $v$ and $w$ in $\mathbb{N}^{n}$ and $0 \leq w_{i}<p$ for every $i$. We deduce

$$
X^{w} \in\left(J_{p}^{\left[p^{e}\right]}: X^{p v}\right)=\left(J_{p}^{\left[p^{e-1}\right]}: X^{v}\right)^{[p]},
$$

where the equality follows from the fact that $\mathbb{F}_{p}\left[X_{1}, \ldots, X_{n}\right]$ is flat over $\mathbb{F}_{p}\left[X_{1}^{p}, \ldots, X_{n}^{p}\right]$. The assumption on $w$ implies that $w_{i}=0$ for every $i$ and $\left(J_{p}^{\left[p^{e-1}\right]}: X^{v}\right)$ is the unit ideal. We deduce that $X^{v}$ lies in $J_{p}^{\left[p^{e-1}\right]}$, hence in $\widetilde{J}_{p}^{\left[p^{e-1}\right]}$ by the induction hypothesis. Therefore $X^{u}$ is in $J_{p}^{\left[p^{e}\right]}$, which completes the proof of the proposition. 


\section{References}

[Bj] J.-E. Björk, Rings of differential operators, Amsterdam, North-Holland, 1979.

[BMS1] N. Budur, M. Mustaţă and M. Saito, Combinatorial description of the roots of the Bernstein-Sato polynomials for monomial ideals, to appear in Comm. Algebra.

[BMS2] _ Bernstein-Sato polynomials of arbitrary varieties, to appear in Compos. Math.

[ELSV] L. Ein, R. Lazarsfeld, K. E. Smith and D. Varolin, Jumping coefficients of multiplier ideals, Duke Math. J. 123 (2004) 469-506.

[HY] N. Hara and K.-i. Yoshida, A generalization of tight closure and multiplier ideals, Trans. Amer. Math. Soc. 355 (2003) 3143-3174.

[Ho1] J. Howald, Multiplier ideals of monomial ideals, Trans. Amer. Math. Soc. 353 (2001), 2665-2671.

[Ka] M. Kashiwara, D-modules and microlocal calculus, translated from the 2000 Japanese original by Mutsumi Saito. Translations of Mathematical Monographs, 217. Iwanami Series in Modern Mathematics. American Mathematical Society, Providence, RI, 2003.

[Ko] J. Kollár, Singularities of pairs, Algebraic geometry—Santa Cruz 1995, 221-287, Proc. Sympos. Pure Math., 62, Part 1, Amer. Math. Soc., Providence, RI, 1997.

[La] R. Lazarsfeld, Positivity in algebraic geometry II, Positivity for vector bundles, and multiplier ideals. A Series of Modern Surveys in Mathematics [Results in Mathematics and Related Areas. 3rd Series. A Series of Modern Surveys in Mathematics], 49. Springer-Verlag, Berlin, 2004.

[MTW] M. Mustaţă, S. Takagi and K.-i. Watanabe, F-thresholds and Bernstein-Sato polynomials, to appear in A. Laptev (ed.), European congress of mathematics (ECM), Stockolm, Sweden, June 27-July 2, 2004, Zürich, European Mathematical Society, 2005.

[Zi] G. Ziegler, Lectures on polytopes, Graduate Texts in Mathematics 152, SpringerVerlag, New York, 1995.

Department of Mathematics, The Johns Hopkins University, Baltimore, MD 21218

E-mail address: budur@math.jhu.edu

Department of Mathematics, University of Michigan, Ann Arbor, Mi 48109

E-mail address: mmustata@umich.edu

RIMS KYoto UnIVERSITY, KYOTO 606-8502, JAPAN

E-mail address: msaito@kurims.kyoto-u.ac.jp 